\title{
Triptolide inhibits human telomerase reverse transcriptase by downregulating translation factors SP1 and c-Myc in Epstein-Barr virus-positive B lymphocytes
}

\author{
CONG LONG ${ }^{1}$, QIU-BO XU ${ }^{1}$, LI DING ${ }^{1}$, LIU YANG ${ }^{1}$, WEI JI ${ }^{1}$, FENG GAO $^{2}$ and YONG JI ${ }^{2}$ \\ ${ }^{1}$ Clinical Laboratory, ${ }^{2}$ Department of General Surgery, Jingjiang People's Hospital, Jingjiang, Jiangsu 214500, P.R. China
}

Received June 9, 2020; Accepted November 17, 2020

DOI: $10.3892 / \mathrm{ol} .2021 .12541$

\begin{abstract}
Epstein-Barr virus (EBV) mainly causes infectious mononucleosis and is associated with several neoplasms, including Burkitt's lymphoma, nasopharyngeal carcinoma and lymphoproliferative disease. Human telomerase reverse transcriptase (hTERT) regulates enzymatic activity of telomerase and is closely associated with tumorigenesis and senescence evasion. Triptolide (TP) is a diterpenoid triepoxide, with a broad-spectrum anticancer and immunosuppressive bioactivity profile. The present study investigated whether TP inhibited hTERT expression and suppressed its activity. The mRNA and protein levels of hTERT were examined by reverse transcription-quantitative PCR and western blotting. The activity of hTERT promoter was determined by dual-luciferase reporter assay. Cell Counting Kit-8 assays were performed to analyze cell proliferation. The present study used EBV-positive B lymphoma cells as a model system, and the results demonstrated that TP significantly decreased hTERT transcription and protein expression. Mechanistically, TP attenuated the hTERT promoter activity by downregulating the expression levels of specificityprotein 1 and c-Myc transcription factors. Consistently, inhibition of hTERT via shRNA transfection efficiently enhanced the suppression of cell proliferation by TP. Furthermore, TP increased virus latent replication and promoted the lytic cycle of EBV in EBV-positive B lymphoma cells, increasing the number of lytic cells and inhibiting the viability of tumor cells. Taken together, the results of the present study revealed a molecular mechanism of the pharmacological inhibition of tumor cell proliferation by TP, encouraging the translation of TP-based therapeutics in EBV-positive B lymphoma treatment.
\end{abstract}

Correspondence to: Dr Feng Gao or Dr Yong Ji, Department of General Surgery, Jingjiang People's Hospital, 28 Zhongzhou East Road, Jingjiang, Jiangsu 214500, P.R. China

E-mail: wwangfirst@163.com

E-mail: jjrmyongji@163.com

Key words: Epstein-Barr virus, triptolide, human telomerase reverse transcriptase, specificity protein $1, \mathrm{c}-\mathrm{Myc}$

\section{Introduction}

Epstein-Barr virus (EBV), a large double-stranded DNA virus, was discovered in 1964 from African Burkitt's lymphoma (BL) cells (1). Like all herpesviruses, EBV can infect cells in either their latent or lytic forms. As a ubiquitous human virus, EBV causes infectious mononucleosis and persists in the individual for life; however, it is normally well controlled by the immune system (2). EBV is also associated with different types of human cancer of both B-cell and epithelial origin, including $\mathrm{BL}$, nasopharyngeal carcinoma, lymphoproliferative disease and gastric cancer $(2,3)$. In addition, increasing evidence has indicated that EBV reactivation occurs in a subset of individuals with autoimmune diseases, including multiple sclerosis and rheumatoid arthritis (4,5). It has been demonstrated that EBV is reactivated under psychological stress, and chronic EBV reactivation is an important mechanism in the pathogenesis of these diseases (4).

Telomeres are located at the ends of chromosomes composed of tandemly repeated G-rich DNA sequences, and they predominantly function to maintain chromosomal integrity and genome stability (6). The progression through replication cycles leads to telomere-dependent pathways of senescence and mortality (7). Telomerase, a complex ribonucleoprotein, resolves this problem by adding TTAGGG repeats to the ends of the chromosomes to promote the capping of eukaryotic telomere ends (8). Human telomerase reverse transcriptase (hTERT), the catalytic subunit of telomerase, plays an important role in the rate-limiting step of activating telomerase (9). Transcriptional regulation is likely to be the main mechanism for regulating hTERT expression (9). hTERT exhibits little or no expression in normal somatic cells; however, it is abundantly present in stem and germ cells (10). Dysregulation of hTERT results in abnormal activation in $~ 85 \%$ of human cancers, including cholangiocarcinoma, breast cancer and gastric cancer (11). Notably, cancer cells have acquired the ability to overcome and enhance tumor growth by maintaining the length and activity of telomere, which makes hTERT an attractive cancer biomarker in clinical practice (12). Recently, novel hTERT-based therapies have been developed, such as immunotherapy and small molecule interfering therapy $(13,14)$. Thus, hTERT has been selected as a target of small molecular drugs in human cancer (13). 
Triptolide (TP), a diterpenoid triepoxide, is extracted from the root of the Chinese herb Tripterygium wilfordii (15). TP possesses a broad-spectrum of bioactivity and cytotoxic functions, including antitumor, anti-inflammatory, anti-fertility and immunosuppressive properties $(16,17)$. TP has been demonstrated to exert a potent anticancer effect that involves multiple signaling pathways, including the $\mathrm{NF}-\kappa \mathrm{B}$ signaling pathway, and targets in different types of cancer, including pancreatic cancer, ovarian cancer, cholangiocarcinoma and non-small cell lung carcinoma (18). TP also suppresses the growth of breast cancer by decreasing HMGB1 expression (19). In addition, inhibiting Pol III transcription with TP provides a new method for developing novel treatments for colorectal cancer (20). TP inhibits the proliferation of prostate cancer cells by decreasing SUMO-Specific Protease 1 expression (21). TP has demonstrated prominent efficacy as an antitumor and anti-inflammatory inhibitor compared with aclacinomycin, and has entered clinical trials for the treatment of diabetic nephropathy and nephritic syndrome (16). Furthermore, good tolerance to a water-soluble pre-drug of TP has been observed in patients with pancreatic cancer (18).

Despite several investigations, the effect of TP on herpesvirus-related malignancies remains unclear $(20,21)$. A previous study demonstrated that TP inhibits the proliferation of EBV-positive B lymphocytes by downregulating the viral protein, LMP1 (22). The results of the present study demonstrated that TP inhibited hTERT transcription and translation in EBV-positive B lymphocytes. In addition, inhibition of hTERT decreased the proliferation of EBV-positive B lymphocytes. Mechanistically, TP inhibits hTERT transcription by downregulating the transcription factors specificityprotein 1 (SP1) and c-Myc, which bind to the promoter domain.

\section{Materials and methods}

Cell lines and reagents. B95-8 and P3HR-1 EBV-positive B lymphoma cell lines were kindly provided by Professor Y. Cao at Central South University (Changsha, China). 293T cells were purchased from the American Type Culture Collection. EBV cells were maintained in RPMI-1640 (HyClone; Cytiva) supplemented with $10 \%$ fetal bovine serum (FBS; Thermo Fisher Scientific, Inc.), while 293T cells were maintained in DMEM (HyClone; Cytiva) supplemented with $10 \% \mathrm{FBS}$ at $37^{\circ} \mathrm{C}$ with $5 \% \mathrm{CO}_{2}$. All cell lines were authenticated via short tandem repeat analysis and cultured for 5 months following resuscitation. TP, cycloheximide (both purchased from Merck KGaA), and cidofovir (CDV; Selleck Chemicals) were dissolved in DMSO.

Plasmids. The pGL3.0 luciferase reporter vector was purchased from Promega Corporation. Renilla plasmid was kindly provided by Professor DeYin Guo from Wuhan University (Wuhan, China). The hTERT promoter regions that span from $-1126,-792,-461$ or -277 to -47 were amplified from the BCBL-1 genome and subsequently inserted into pGL3.0 (23). All constructs were purified using the Plasmid Miniprep kit (cat. no. AP-MN-P-250; Corning Inc.), according to the manufacturer's instructions.

Cell transfection.Atotal of $4 \times 10^{5}$ cells/well were placed in 6-well plates. B95-8 and P3HR-1 cells were transiently transfected with $0.5 \mu \mathrm{g} / \mathrm{ml}$ hTERT shRNA (5'-GAACTTCCCTGTAGA AGACGA-3') or $0.5 \mu \mathrm{g} / \mathrm{ml}$ non-targeting control shRNA (5'-TACAACAGCCACAACGTCTAT-3') (both Shanghai GenePharma, Co., Ltd.) using X-tremeGENE HP DNA Transfection Reagent (Roche Diagnostics), according to the manufacturer's protocol. All cells were maintained in RPMI-1640 medium supplemented with $10 \% \mathrm{FBS}$ at $37^{\circ} \mathrm{C}$ for $48 \mathrm{~h}$. At $48 \mathrm{~h}$ post-transfection, cells were collected for subsequent experiments.

Dual-luciferase reporter assay. Dual-luciferase reporter assay was performed as described previously (23). Briefly, each luciferase reporter construct or pGL3.0 $(0.5 \mu \mathrm{g})$ and pRL-TK $(0.05 \mu \mathrm{g})$ (kindly provided by Professor D. Guo of Wuhan University) was co-transfected into 293T cells in triplicates, using Lipofectamine ${ }^{\circledR} 2000$ (Thermo Fisher Scientific, Inc.) for $24 \mathrm{~h}$ at $37^{\circ} \mathrm{C}$. Cells were incubated at $37^{\circ} \mathrm{C}$ with TP $(200 \mathrm{nM})$ or control (DMSO) for a further $24 \mathrm{~h}$. Following incubation, luciferase activities were detected using the Dual-Luciferase Assay kit (cat. no. E1960; Promega Corporation), according to the manufacturer's instructions. The activity of firefly luciferase was normalized to Renilla luciferase control values and is shown as an average of triplicates.

Immunoblotting. Immunoblotting was performed as previously described (24). Briefly, B95-8 and P3HR-1 cells were lysed using RIPA buffer (Beyotime Institute of Biotechnology) supplemented with $0.5 \%$ proteasome inhibitor cocktail (Roche Diagnostics). Protein concentrations were determined using the Bradford Protein Assay kit (cat. no. 5000201; Bio-Rad Laboratories, Inc.) and 30-50 $\mu \mathrm{g}$ protein/lane was separated via $15 \%$ SDS-PAGE. The separated proteins were subsequently transferred onto PVDF membranes (Bio-Rad Laboratories, Inc.) and blocked with $5 \%$ fat-free milk for $1 \mathrm{~h}$ at room temperature. The membranes were incubated with primary antibodies against GAPDH (1:10,000; cat. no. 10494-1-AP; ProteinTech Group, Inc.), SP1 (1:1,000; cat. no. 9389; Cell Signaling Technology, Inc.) and hTERT (1:1,000; cat. no. sc-7212; Santa Cruz Biotechnology, Inc.) overnight at $4^{\circ} \mathrm{C}$. Following the primary incubation, membranes were incubated with HRP-conjugated anti-rabbit IgG secondary antibodies (1:10,000; cat. no. sc-2357; Santa Cruz Biotechnology, Inc.) for $1 \mathrm{~h}$ at room temperature, and protein bands were detected using SuperSignal Chemiluminescent Substrate (Bio-Rad Laboratories, Inc.).

Cell viability assay. B95-8 and P3HR-1 cells transfected with hTERT shRNA were seeded at a density of 20,000 cells/well into 96-well plates and maintained in RPMI-1640 media supplemented with $10 \% \mathrm{FBS}$ at $37^{\circ} \mathrm{C}$ for $24 \mathrm{~h}$. Cells were subsequently treated with TP $(200 \mathrm{nM})$ at $37^{\circ} \mathrm{C}$ for $24 \mathrm{~h}$. Cell viability was assessed via the Cell Counting Kit-8 (CCK-8; Dojindo Molecular Technologies, Inc.) assay by incubating cells with the CCK-8 reagent for $30 \mathrm{~min}$ in the dark at $37^{\circ} \mathrm{C}$, according to the manufacturer's instructions. The optical density was detected at a wavelength of $450 \mathrm{~nm}$ using a microplate reader (BioTek China).

Analysis of hTERT degradation. B95-8 and P3HR-1 cells were treated with TP $(0$ or $200 \mathrm{nM})$ for $24 \mathrm{~h}$, and Cycloheximide (CHX; $25 \mathrm{mg} / \mathrm{ml}$; cat. no. C7698; Sigma-Aldrich; 
Merck KGaA) for a further $12 \mathrm{~h}$ at $37^{\circ} \mathrm{C}$ before harvest. hTERT protein expression was detected via immunoblotting, as aforementioned. GAPDH was used as the loading control.

In silico analysis. To identify potential transcription factors regulating hTERT expression, in silico analysis was performed using the University of California Santa Cruz (UCSC) genome browser gateway (http://genome.ucsc.edu/) for the human genomic information of GRCh38/hg38. Potential transcription factors and binding regions were obtained in the ChIP-seq clusters.

Progeny virus analysis. 12-O-Tetradecanoylphorbol-13Acetate (TPA; $20 \mathrm{ng} / \mathrm{ml})$ and sodium butyrate $(\mathrm{NaB} ; 1.2 \mathrm{mM})$ were used to induce B95-8 and P3HR-1 cells entering lytic replication. After $3 \mathrm{~h}$ of induction at $37^{\circ} \mathrm{C}$, the cell medium was replaced and cells were cultured in the absence or presence of TP $(0,100$ or $200 \mathrm{nM})$ and CDV $(20 \mu \mathrm{M})$ for another $24 \mathrm{~h}$ at $37^{\circ} \mathrm{C}$. EBV virion-associated DNA derived from the B95-8 and P3HR-1 cell supernatants was collected as previously described (25). Equal amounts of viral lysate were used for reverse transcription-quantitative PCR (RT-qPCR) of the EBV specific region, EBNA1. Intracellular viral DNA of B95-8 and P3HR-1 cells was extracted and evaluated by RT-qPCR analysis.

RT-qPCR. Total cellular RNA was extracted from B95-8 and P3HR-1 cells using TRIzol ${ }^{\circledR}$ reagent (Thermo Fisher Scientific, Inc.). RT-qPCR was performed as previously described (25). Briefly, total RNA was reverse transcribed into cDNA using the Reverse Transcription kit (cat. no. 639505; Takara Bio, Inc.) according to the manufacturer's instructions, and quantified using the CFX 96 Real-Time PCR Detection System (Bio-Rad Laboratories, Inc.). The primer sequences used for qPCR were as previously described (23). Relative mRNA expression levels were calculated using the $2^{-\Delta \Delta C q}$ method (26) and normalized to the internal reference gene GAPDH.

Statistical analysis. All experiments were repeated for three independent cultures. Statistical analysis was performed using GraphPad Prism 5 (GraphPad Software, Inc.). Unpaired two-tailed Student's t-test was used to compare differences between two groups, while two-way ANOVA and Tukey's post hoc test were used to compare differences between multiple groups. $\mathrm{P}<0.05$ was considered to indicate a statistically significant difference.

\section{Results}

TP decreases hTERT transcription and protein expression in EBV-positive B lymphoma cells. TP has previously been demonstrated to decrease hTERT expression in primary effusion lymphoma (PEL) cells (23). To determine whether TP plays a similar role in EBV-positive B lymphoma cells, immunoblotting was performed in B95-8 and P3HR-1 cells treated with $\mathrm{TP}$ in a dose-dependent manner. The results demonstrated that TP significantly downregulated hTERT expression in B95-8 (Fig. 1A) and P3HR-1 (Fig. 1B) cells. Furthermore, B95-8 and P3HR-1 cells were treated with TP in the presence or absence of CHX to determine whether TP inhibits hTERT expression through hTERT half-time. As presented in Fig. 1C and D, the stability of hTERT decreased in both B95-8 and P3HR-1 cells in the presence of TP. These results indicated that TP mainly attenuated hTERT protein expression, and partly the level of protein stability. Mechanistically, RT-qPCR analysis was performed in B95-8 and P3HR-1 cells treated with TP in a dose-dependent manner to detect whether decreased hTERT expression affected mRNA levels. As presented in Fig. 1E and $\mathrm{F}$, treatment with TP significantly decreased hTERT mRNA levels in both B95-8 and P3HR-1 cells, particularly following the administration of $200 \mathrm{nM}$ TP. Taken together, these results suggest that TP significantly inhibits mRNA and protein expression of hTERT in EBV-positive B lymphoma cells.

TP inhibits the promoter activity of hTERT. Dysregulation of hTERT occurs in $\sim 85 \%$ of human cancers, with transcriptional regulation likely to be the major mechanism regulating hTERT expression (11). To determine whether TP affects the activity of the hTERT promoter, serial truncations of hTERT promoter domains were inserted into the pGL3.0 vector (Fig. 2A). These constructs were subsequently transfected into $293 \mathrm{~T}$ cells with Renilla, followed by treatment with TP $24 \mathrm{~h}$ post-transfection. The promoter activities were determined using the Dual-GLU system after $24 \mathrm{~h}$. As presented in Fig. 2B, treatment with TP significantly decreased the luciferase activities of hTERT promoters, $-1126 /-47,-792 /-47$ and $-461 /-47$, and particularly that of the hTERT promoter -277/-47. Collectively, these results suggest that the promoter domain, $-277 /-47$ is likely to be the region most responsive to TP.

TP decreases expression of the transcription factors, SPI and c-Myc in EBV-positive B lymphoma cells. Transcription factor SP1, a member of the zinc finger family, is considered a basal transcription factor that regulates housekeeping genes (27). A previous study demonstrated that the hTERT promoter sequence contains several SP1 binding motifs (GC boxes), and plays an important role in upregulating Kaposi's sarcoma-associated herpesvirus-encoded latency-associated nuclear antigen (28). In addition, the super-transcription factor c-Myc potentially regulates the transcription of at least $15 \%$ of the entire genome (29). Thus, in silico analysis was performed in the UCSC genome browser gateway for the human genomic information of GRCh38/hg38, and the results demonstrated that the hTERT promoter sequence contains the SP1 and c-Myc binding domains (Fig. 2C). To identify the effect of TP on SP1, c-Myc, and Mad1, B95-8 and P3HR-1 cells were treated with TP $(0,100$ or $200 \mathrm{nM})$ for $24 \mathrm{~h}$. As presented in Fig. 2D, treatment of B95-8 and P3HR-1 cells with TP in a dose-dependent manner slightly enhanced Mad1 expression, and notably decreased SP1 and c-Myc expression levels. Taken together, these results suggest that TP downregulates SP1 and c-Myc expression in B95-8 and P3HR-1 cells.

$T P$ increases lytic and latent replication of $E B V$ in EBV-positive B lymphoma cells. To determine the effect of TP on the antiviral activity, B95-8 and P3HR-1 cells uninduced or induced by TPA $(20 \mathrm{ng} / \mathrm{ml})$ and $\mathrm{NaB}(1.2 \mathrm{mM})$ for $3 \mathrm{~h}$ were treated with TP $(0,100$ or $200 \mathrm{nM})$. After $24 \mathrm{~h}$, supernatants, cell lysates and genomic DNA were prepared and incubated for a further $48 \mathrm{~h}$. RT-qPCR analysis was performed to 
A
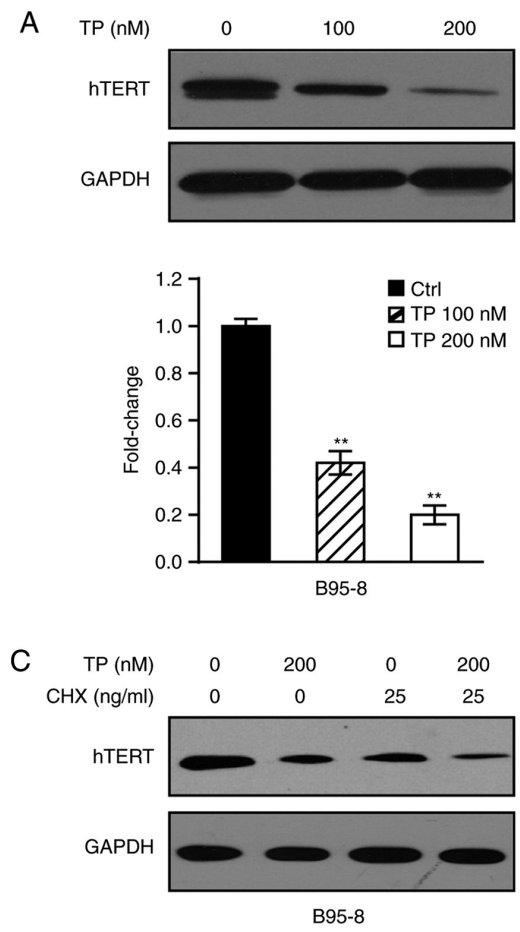

E

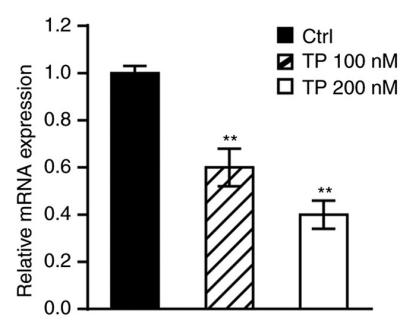

B
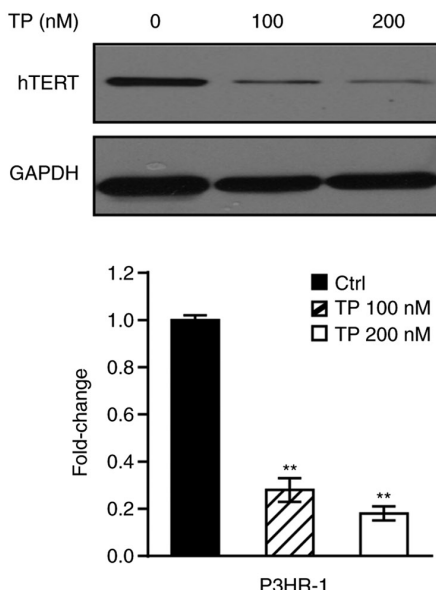

D

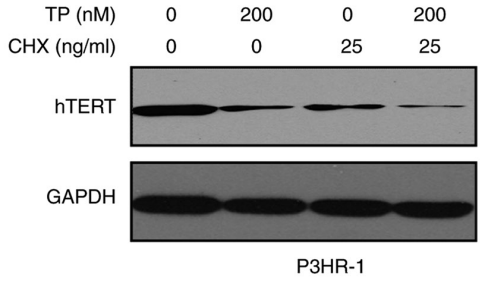

$\mathrm{F}$

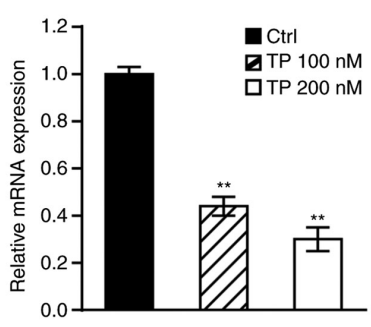

P3HR-1

Figure 1. TP attenuates hTERT protein accumulation and transcriptional activity. Immunoblots (above) and quantitation (below) of hTERT in (A) B95-8 and (B) P3HR-1 cells, with or without TP treatment for $24 \mathrm{~h}$. GAPDH was used as the loading control. (C) B95-8 and (D) P3HR-1 cells were treated with TP $(0$ or $200 \mathrm{nM})$ for $24 \mathrm{~h}$, and CHX $(25 \mathrm{mg} / \mathrm{ml})$ for a further $12 \mathrm{~h}$ before harvest. hTERT protein expression was detected via immunoblotting. GAPDH was used as the loading control. (E) B95-8 and (F) P3HR-1 cells were treated with TP $(0,100$ or $200 \mathrm{nM})$ for $24 \mathrm{~h}$. hTERT mRNA expression was detected via reverse transcription-quantitative PCR analysis. ${ }^{* *} \mathrm{P}<0.01$ vs. Ctrl. TP, triptolide; hTERT, human telomerase reverse transcriptase; Ctrl, control; CHX, cycloheximide.

A

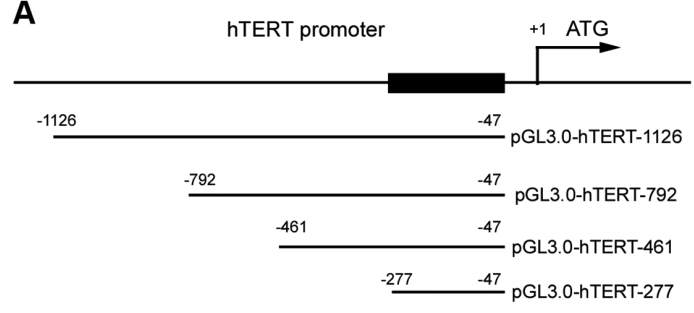

C

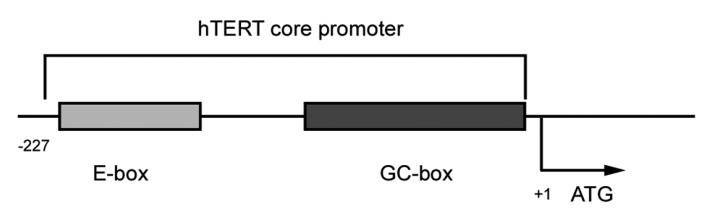

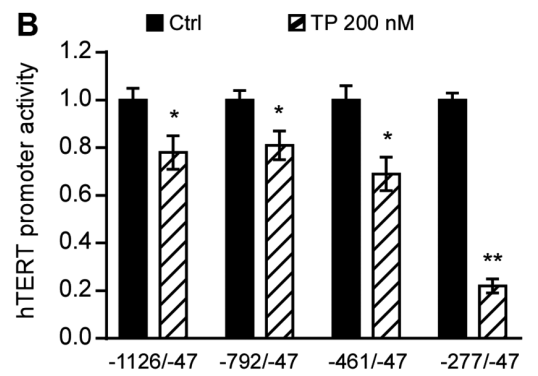

D

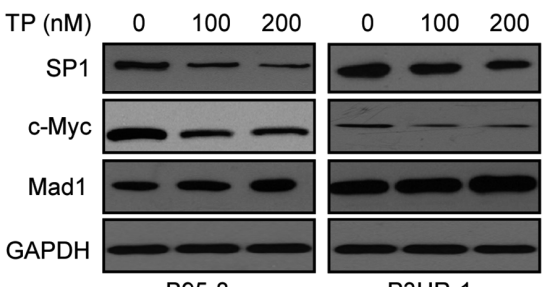

B95-8

P3HR-1

Figure 2. TP decreases SP1 expression. (A) Schematic diagram of hTERT promoter and truncations. (B) 293T cells were co-transfected with Renilla and the indicated hTERT promoter constructs, followed by treatment with TP $(0$ or $200 \mathrm{nM})$ for $24 \mathrm{~h}$ post-transfection. Luciferase reporter activities were measured using the Dual Luciferase Assay kit. (C) Schematic diagram of the hTERT core promoter. (D) Immunoblotting was performed to detect SP1, c-Myc and Mad1 protein expression levels in B95-8 and P3HR-1 cells, with or without TP $(0,100$ or $200 \mathrm{nM})$ for $24 \mathrm{~h}$. GAPDH was used as the loading control. "P<0.05 and ${ }^{* *} \mathrm{P}<0.01$ vs. Ctrl. TP, triptolide; SP1, specificityprotein 1; hTERT, human telomerase reverse transcriptase; Ctrl, control. 

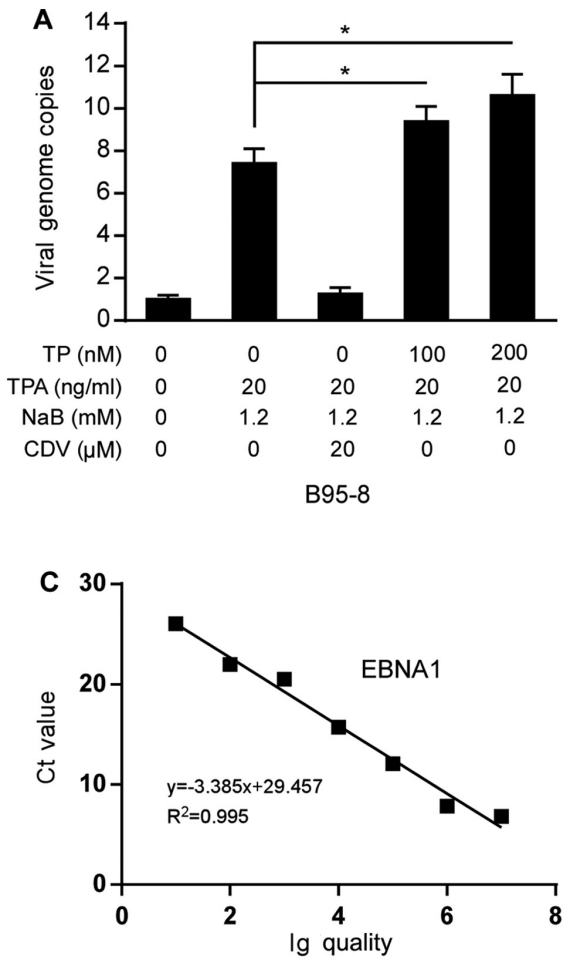

E

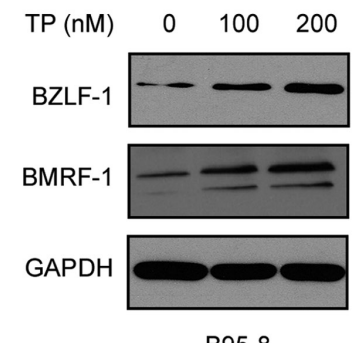

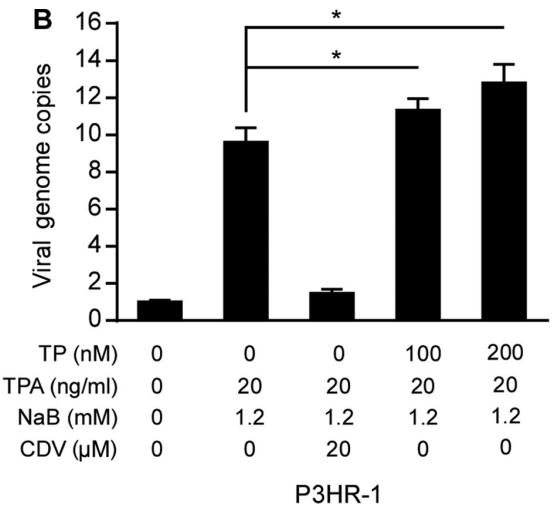

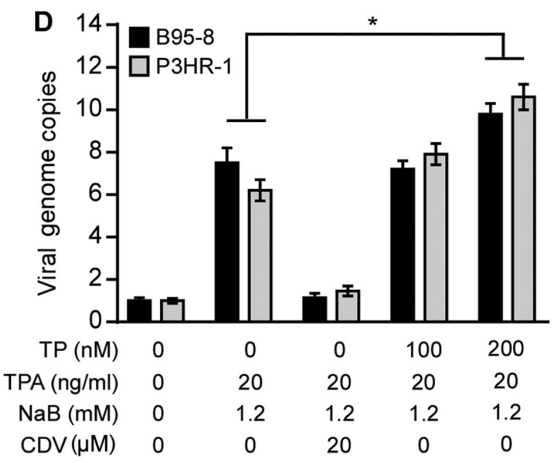

$\mathbf{F}$

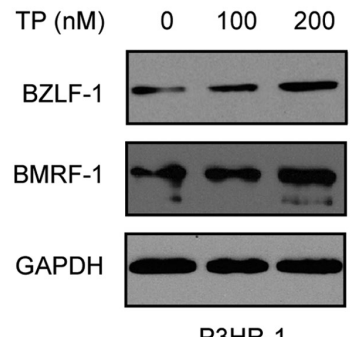

P3HR-1

Figure 3. TP promotes the virus production and lysis of EBV in B95-8 and P3HR-1 cells. Uninduced and induced (A) B95-8 and (B) P3HR-1 cells were treated with TP for $24 \mathrm{~h}$, and cell supernatants, genomic DNA and lysates were prepared. The copy numbers of the intracellular viral DNA were detected via RT-qPCR analysis and normalized to the internal reference gene GAPDH. (C) Standard curve for virions quantification used to calculate the number of EBV virions in the supernatants of B95-8 and P3HR-1 cells. (D) Virion production extracted from equivalent B95-8 and P3HR-1 cell supernatants was determined by RT-qPCR, and the viral copy numbers were calculated using the standard curve derived from the EBNA1 construct. Immunoblotting was performed to detect BZLF-1 and BMRF-1 protein expression following treatment of (E) B95-8 and (F) P3HR-1 cells with TP (0,100 or $200 \mathrm{nM})$ for $24 \mathrm{~h}$. GAPDH was used as the loading control. "P<0.05. TP, triptolide; EBV, Epstein-Barr virus; RT-qPCR, reverse transcription-quantitative PCR; TPA, 12-O-Tetradecanoylphorbol-13-Acetate; $\mathrm{NaB}$, sodium butyrate; $\mathrm{CDV}$, cidofovir.

detect the lytic and latent replication of EBV. As presented in Fig. 3A and B, treatment with TP increased the number of viral genome copies in B95-8 and P3HR-1 cells. Production of progeny virion extracted from the B95-8 and P3HR-1 cell supernatants was calculated according to the standard curve derived from the EBNA1 construct (Fig. 3C). Results indicated that the production of progeny virion was increased after TP treatment in B95-8 and P3HR-1 cell supernatants (Fig. 3D). CDV, a positive control, efficiently decreased the lytic replication of EBV, as previously described (30). Similar to the effect of TP on intracellular production of progeny virion, extracellular production of progeny virion was also increased in induced B95-8 and P3HR-1 cells (Fig. 3D). On the contrary, CDV decreased intracellular and extracellular production of progeny virion in induced B95-8 and P3HR-1 cells.
The transition from the latent to the lytic state is triggered by two viral transcription factors, BZLF-1 (also known as ZEBRA, Zta or EB1) and BMRF-1 (also known as Rta or R) (31). The promoters regulating BZLF-1 and BMRF-1 expression are tightly repressed during latency (30). It was speculated that TP promotes the entry of EBVs into the lytic period. Thus, B95-8 and P3HR-1 cells were treated with TP $(0,100$ or $200 \mathrm{nM})$ for $24 \mathrm{~h}$ and harvested for immunoblotting analysis. As presented in Fig. $3 \mathrm{E}$ and $\mathrm{F}$, treatment with $\mathrm{TP}$ markedly increased BZLF-1 and BMRF-1 expression in B95-8 and P3HR-1 cells. Collectively, these results suggest that TP promotes the lytic state, and increases the production of EBV progeny virions in EBV-positive B lymphoma cells.

Inhibition of hTERT enhances the suppression of TP on the proliferation of EBV-positive B lymphoma cells. To determine 

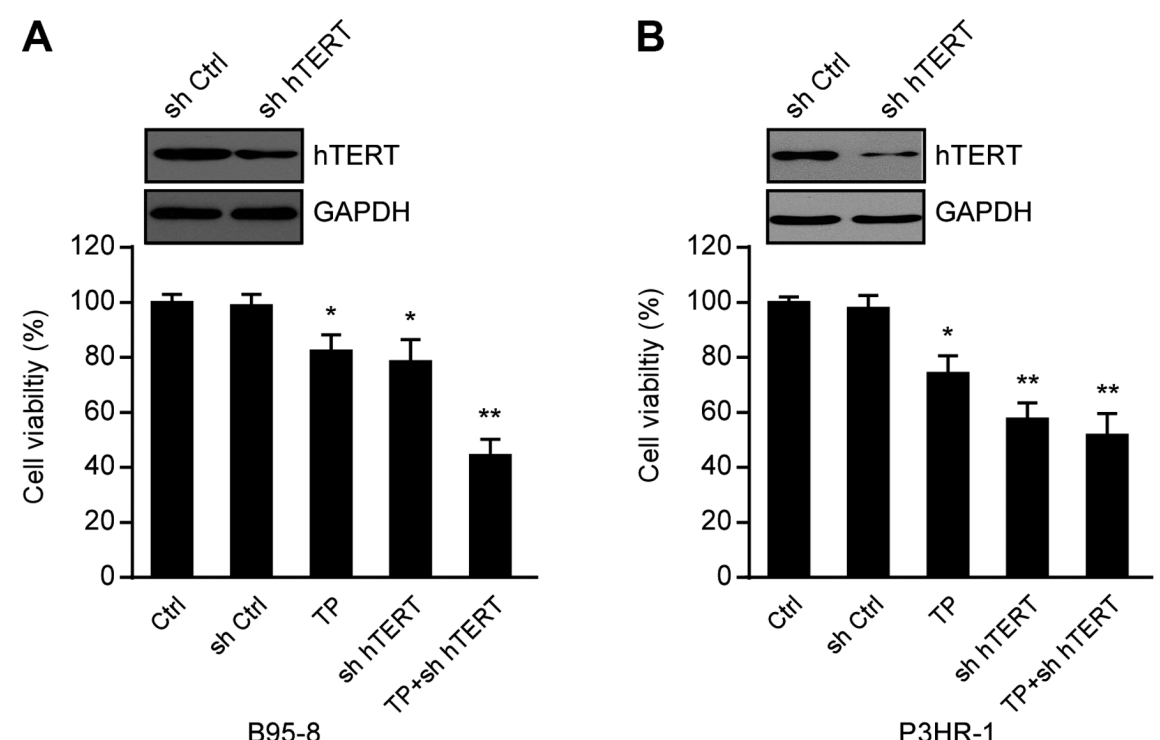

Figure 4. hTERT inhibition attenuates cell viability. (A) B95-8 and (B) P3HR-1 cells, transfected with Ctrl or specific sh hTERT for $48 \mathrm{~h}$ and subjected to western blotting, were treated with TP $(0$ or $200 \mathrm{nM})$ for another $24 \mathrm{~h}$. Cell viability was assessed via the CCK-8 assay. "P<0.05 and ${ }^{* *} \mathrm{P}<0.01$ vs. Ctrl. hTERT, human telomerase reverse transcriptase; TP, triptolide; Ctrl, control; sh, short hairpin.

the effect of hTERT inhibition and TP on cell viability, B95-8 and P3HR-1 cells transfected with or without hTERT shRNA and control shRNA were treated with TP $(0$ and $200 \mathrm{nM})$. After $24 \mathrm{~h}$, cell viability was assessed via the CCK- 8 assay. As presented in Fig. 4A and B, both TP- and hTERT-knockdown inhibited B95-8 and P3HR-1 cell viability, respectively. In addition, the combination of TP- and hTERT-knockdown synergistically decreased cell viability. Taken together, these results suggest that TP- and hTERT-knockdown inhibit cell proliferation, particularly when combined.

\section{Discussion}

Telomerase reactivation is a critical hallmark of $>90 \%$ of cancers (12). However, the molecular mechanism regulating hTERT expression in tumors remains elusive. Thus, understanding how hTERT is activated and how it contributes to tumor continues to be a major research focus. The present study used EBV-positive B lymphoma cells as a model system, and the results confirmed that TP prominently inhibited hTERT at the transcriptional and translational levels in vitro. Mechanistically, the present study suggested that TP decreased the expression levels of the transcription factors, SP1 and c-Myc in EBV-positive B lymphoma cells. Notably, the results of the present study demonstrated that TP increased the lytic and latent replication of EBV in B95-8 and P3HR-1 cells, suggesting that TP may be effective as an hTERT-selective cancer therapeutic.

hTERT expression is typically detectable in cancerous tissues and immortalized cells (13). KSHV-encoded LANA1 has been demonstrated to enhance the transactivation of hTERT promoter by interacting with SP1 (28). A previous study demonstrated that TP decreases LANA1 expression in KSHV- and herpesvirus-related PEL cells (25). Another study reported that TP inhibits the transcription of hTERT by downregulating SP1 in PEL cells (23). The results of the present study demonstrated that hTERT knockdown inhibited cell viability, particularly in combination with TP, which expanded the therapeutic tumor types. Furthermore, the results of the promoter truncation assay indicated that TP decreased the luciferase activity of a serial truncation hTERT promoter construct. This mechanism suggested that the hTERT promoter activity inhibited by TP led to a reduction in the transcription levels of hTERT, particularly in the -277/-47 promoter domain.

The -277/-47 promoter domain of hTERT contains five $\mathrm{GC}$ boxes, which are binding sites for zinc finger transcription factor SP1 (32). As a basal transcription factor, SP1 recognizes GC boxes and regulates a series of signaling pathways by interacting with other transcriptional activators (27). Thus, SP1 binds to the GC boxes and functions synergistically to maintain the activity of hTERT. In the present study, the pharmacological inhibition of SP1 by TP decreased hTERT expression. In addition, as a super-transcription factor, Myc potentially regulates the transcription of at least $15 \%$ of the entire genome, and is deregulated in $>50 \%$ of human cancers $(29,33)$. Previous studies have demonstrated that c-Myc acts as a key regulator of hTERT during tumorigenesis by binding to the E-box $(11,34)$. In the present study, TP significantly deceased c-Myc protein expression, partially accounting for the attenuation of hTERT activity in TP treatment. It has been reported that hTERT activity and expression is associated with the $\mathrm{Myc} / \mathrm{Mad} / \mathrm{Max}$ network in colon cancer cells (35). Conversely, in the present study the expression of Mad1, a transcriptional repressor of hTERT activity, was not affected by treatment with TP in EBV-positive B lymphoma cells. The hTERT core promoter is highly specific to cancer cells, and there may be other transcription factors that are affected by TP, leading to the inhibition of hTERT expression.

EBV has two modes of infection, latent and lytic (36). EBV, with a predominantly latent form, infects almost all humans during their lifetime, and following the lytic phase, persists for the remainder of the individuals life (37). The transition from the latent to the lytic state is regulated by the expression of two 
transcription factors, BZLF-1 and BRLF-1 $(31,38)$. BZLF-1 is the master regulator of the EBV lytic cycle, whereby overexpression of BZLF-1 in latent cells can initiate the lytic cycle and drive it to completion (39). In addition, the primary role of BZLF-1 is to stimulate the BRLF-1 promoter during lytic reactivation (40). Given that most cells in EBV-associated tumors harbor latent virus, several studies have attempted to reactivate the lytic cycle for realizing oncolytic treatment repertoire $(41,42)$. Thus, BZLF-1 and BRLF-1 were selected and analyzed in the present study. TP inhibition of interferon production sensitizes prostate cancer PC3 cells to vesicular stomatitis virus replication and virus-mediated apoptosis (43). The results of the present study demonstrated that TP increases latent replication and promotes the lytic cycle of EBV in EBV-positive B lymphoma cells, improving the number of lytic reactivated cells and inhibiting cell viability. Overall, the results of the present study suggest that TP-based therapeutics may be used to treat EBV-positive B lymphoma. It was demonstrated that TP inhibited hTERT by downregulating the transcription factors SP1 and c-Myc in EBV-positive $\mathrm{B}$ lymphocytes. The toxicity of TP in vivo and other mechanisms induced by TP treatment should be further investigated in EBV-positive B lymphocytes in future studies.

\section{Acknowledgements}

Not applicable.

\section{Funding}

The present study was supported by the Natural Science Foundation of Hubei Province (grant no. 2017CFB738 awarded to CL).

\section{Availability of data and materials}

The datasets used and/or analyzed during the current study are available from the corresponding author on reasonable request.

\section{Authors' contributions}

FG and YJ conceived and designed the present study. CL and FG supervised the present study. CL and YJ drafted the initial manuscript. CL, QBX and LD performed most of the experiments. CL, QBX, LY, WJ, FG and YJ analyzed the data. LY and WJ provided technical assistance for immunoblotting experiments. All authors have read and approved the final manuscript.

\section{Ethics approval and consent to participate}

Not applicable.

\section{Patient consent for publication}

Not applicable.

\section{Competing interests}

The authors declare that they have no competing interests.

\section{References}

1. Epstein MA, Achong BG and Barr YM: Virus particles in cultured lymphoblasts from burkitt's lymphoma. Lancet 1: 702-703, 1964.

2. Farrell PJ: Epstein-barr virus and cancer. Annu Rev Pathol 14: 29-53, 2019.

3. Klein G: Tumor associations of EBV-historical perspectives. Curr Top Microbiol Immunol 390: 17-22, 2015.

4. Niller HH, Wolf H and Minarovits J: Regulation and dysregulation of Epstein-Barr virus latency: Implications for the development of autoimmune diseases. Autoimmunity 41: 298-328, 2008.

5. Cohen JI: Primary immunodeficiencies associated with EBV disease. Curr Top Microbiol Immunol 390: 241-265, 2015.

6. Blackburn EH: Switching and signaling at the telomere. Cell 106: 661-673, 2001.

7. Campisi J, Kim SH, Lim CS and Rubio M: Cellular senescence, cancer and aging: The telomere connection. Exp Gerontol 36: 1619-1637, 2001.

8. Zvereva MI, Shcherbakova DM and Dontsova OA: Telomerase: Structure, functions, and activity regulation. Biochemistry (Mosc) 75: 1563-1583, 2010.

9. Gladych M, Wojtyla A and Rubis B: Human telomerase expression regulation. Biochem Cell Biol 89: 359-376, 2011.

10. Wright WE, Piatyszek MA, Rainey WE, Byrd W and Shay JW: Telomerase activity in human germline and embryonic tissues and cells. Dev Genet 18: 173-179, 1996.

11. Kyo S, Takakura M, Fujiwara T and Inoue M: Understanding and exploiting hTERT promoter regulation for diagnosis and treatment of human cancers. Cancer Sci 99: 1528-1538, 2008.

12. Jafri MA, Ansari SA, Alqahtani MH and Shay JW: Roles of telomeres and telomerase in cancer, and advances in telomerasetargeted therapies. Genome Med 8: 69, 2016.

13. Lü MH, Liao ZL, Zhao XY, Fan YH, Lin XL, Fang DC, Guo H and Yang SM: hTERT-based therapy: A universal anticancer approach (Review). Oncol Rep 28: 1945-1952, 2012.

14. Mizukoshi E and Kaneko S: Telomerase-targeted cancer immunotherapy. Int J Mol Sci 20: 1823, 2019.

15. Kupchan SM, Court WA, Dailey RG Jr, Gilmore CJ and Bryan RF: Triptolide and tripdiolide, novel antileukemic diterpenoid triepoxides from Tripterygium wilfordii. J Am Chem Soc 94: 7194-7195, 1972.

16. Wong KF, Yuan Y and Luk JM: Tripterygium wilfordii bioactive compounds as anticancer and anti-inflammatory agents. Clin Exp Pharmacol Physiol 39: 311-320, 2012.

17. Hou W, Liu B and Xu H: Triptolide: Medicinal chemistry, chemical biology and clinical progress. Eur J Med Chem 176: 378-392, 2019.

18. Noel P, Von Hoff DD, Saluja AK, Velagapudi M, Borazanci E and Han H: Triptolide and its derivatives as cancer therapies. Trends Pharmacol Sci 40: 327-341, 2019.

19. Jiang W, Chen M, Xiao C, Yang W, Qin Q, Tan Q, Liang Z, Liao X, Mao A and Wei C: Triptolide suppresses growth of breast cancer by targeting HMGB1 in vitro and in vivo. Biol Pharm Bull 42: 892-899, 2019.

20. Liang X, Xie R, Su J, Ye B, Wei S, Liang Z, Bai R, Chen Z, Li Z and Gao X: Inhibition of RNA polymerase III transcription by Triptolide attenuates colorectal tumorigenesis. J Exp Clin Cancer Res 38: 217, 2019.

21. Huang W, He T, Chai C, Yang Y, Zheng Y, Zhou P, Qiao X, Zhang B, Liu Z, Wang J, et al: Triptolide inhibits the proliferation of prostate cancer cells and down-regulates SUMO-specific protease 1 expression. PLoS One 7: e37693, 2012.

22. Zhou H, Guo W, Long C, Wang H, Wang J and Sun X: Triptolide inhibits proliferation of Epstein-Barr virus-positive B lymphocytes by down-regulating expression of a viral protein LMP1. Biochem Biophys Res Commun 456: 815-820, 2015.

23. Long C, Wang J, Guo W, Wang H, Wang C, Liu Y and Sun X: Triptolide inhibits transcription of hTERT through down-regulation of transcription factor specificityprotein 1 in primary effusion lymphoma cells. Biochem Biophys Res Commun 469: 87-93, 2016.

24. Wang J, Jiang J, Chen H, Wang L, Guo H, Yang L, Xiao D, Qing $\mathrm{G}$ and Liu H: FDA-approved drug screen identifies proteasome as a synthetic lethal target in MYC-driven neuroblastoma. Oncogene 38: 6737-6751, 2019.

25. Long C, Guo W, Zhou H, Wang J, Wang H and Sun X: Triptolide decreases expression of latency-associated nuclear antigen 1 and reduces viral titers in Kaposi's sarcoma-associated and herpesvirus-related primary effusion lymphoma cells. Int J Oncol 48: 1519-1530, 2016. 
26. Livak KJ and Schmittgen TD: Analysis of relative gene expression data using real-time quantitative PCR and the 2(-Delta Delta C(T)) Method. Methods 25: 402-408, 2001.

27. Beishline $\mathrm{K}$ and Azizkhan-Clifford J: Sp1 and the 'hallmarks of cancer'. FEBS J 282: 224-258, 2015.

28. Verma SC, Borah S and Robertson ES: Latency-associated nuclear antigen of Kaposi's sarcoma-associated herpesvirus up-regulates transcription of human telomerase reverse transcriptase promoter through interaction with transcription factor Sp1. J Virol 78: 10348-10359, 2004.

29. Dang CV, O'Donnell KA, Zeller KI, Nguyen T, Osthus RC and Li F: The c-Myc target gene network. Semin Cancer Biol 16: 253-264, 2006.

30. Pica F, Serafino A, Garaci E and Volpi A: Cidofovir on HHV-8 in BCBL-1 cells. Antivir Ther 9: 823-825, 2004.

31. Miller G, El-Guindy A, Countryman J, Ye J and Gradoville L: Lytic cycle switches of oncogenic human gammaherpesviruses. Adv Cancer Res 97: 81-109, 2007.

32. Zhang Y, Sun M, Shi W, Yang Q, Chen C, Wang Z and Zhou X: Arsenic trioxide suppresses transcription of hTERT through down-regulation of multiple transcription factors in HL-60 leukemia cells. Toxicol Lett 232: 481-489, 2015.

33. Meyer N and Penn LZ: Reflecting on 25 years with MYC. Nat Rev Cancer 8: 976-990, 2008.

34. Cong YS, Wen J and Bacchetti S: The human telomerase catalytic subunit hTERT: Organization of the gene and characterization of the promoter. Hum Mol Genet 8: 137-142, 1999.

35. Toaldo C, Pizzimenti S, Cerbone A, Pettazzoni P, Menegatti E, Daniela B, Minelli R, Giglioni B, Dianzani MU, Ferretti C and Barrera G: PPARgamma ligands inhibit telomerase activity and hTERT expression through modulation of the Myc/Mad/Max network in colon cancer cells. J Cell Mol Med 14: 1347-1357, 2010.

36. Tsurumi T, Fujita M and Kudoh A: Latent and lytic Epstein-Barr virus replication strategies. Rev Med Virol 15: 3-15, 2005.
37. Kerr JR: Epstein-Barr virus (EBV) reactivation and therapeutic inhibitors. J Clin Pathol 72: 651-658, 2019.

38. Kanda T: EBV-encoded latent genes. Adv Exp Med Biol 1045 377-394, 2018

39. McKenzie J and El-Guindy A: Epstein-barr virus lytic cycle reactivation. Curr Top Microbiol Immunol 391: 237-261, 2015.

40. Bhende PM, Seaman WT, Delecluse HJ and Kenney SC: BZLF1 activation of the methylated form of the BRLF1 immediate-early promoter is regulated by BZLF1 residue 186. J Virol 79: 7338-7348, 2005.

41. Tikhmyanova N, Paparoidamis N, Romero-Masters J, Feng X, Mohammed FS, Reddy PAN, Kenney SC, Lieberman PM and Salvino JM: Development of a novel inducer for EBV lytic therapy. Bioorg Med Chem Lett 29: 2259-2264, 2019.

42. Murata T and Tsurumi T: Switching of EBV cycles between latent and lytic states. Rev Med Virol 24: 142-153, 2014.

43. Ben Yebdri F, Van Grevenynghe J, Tang VA, Goulet ML, Wu JH, Stojdl DF, Hiscott J and Lin R: Triptolide-mediated inhibition of interferon signaling enhances vesicular stomatitis virus-based oncolysis. Mol Ther 21: 2043-2053, 2013.

(C) $€$ This work is licensed under a Creative Commons Attribution-NonCommercial-NoDerivatives 4.0 International (CC BY-NC-ND 4.0) License. 